\title{
Research of Sign language synthesis Based on VRML
}

\author{
Shicai $\mathrm{Yu}$ \\ College of computer and communication \\ Lanzhou University of Technology \\ Lanzhou, China \\ E-mail: yusc@lut.cn
}

\author{
Rong Lu \\ College of computer and communication \\ Lanzhou University of Technology \\ Lanzhou, China \\ E-mail:1r616@163.com
}

\begin{abstract}
Sign language is to help the deaf and normal hearing people natural communication and computer assisted instruction. Through the analysis of language features, and proposed one kind based on the VRML human body modeling and virtual human based on context of gesture smoothing algorithm, thus the sign language synthesis research and implementation.
\end{abstract}

Keywords- sign language synthesis; synthesis of virtual human; VRML; contextual correlates of gesture motion smoothing algorithm

\section{INTRODUCTION}

China is the most populous country in the world, including a large number of the aural disabled peoples .because of existing the language barrier, it is difficult for the deaf to communicate and then the sign language ,meeting the disabled communication need ,is a kind of special language are gradually accepted by aural disabled peoples. The sign language, a kind of human movement language can not form a relatively stable expression system through the hand movement of semantic expression and a sign of the hand type auxiliary facial expressions and posture. But also, as a main tool for the deaf to make communication and the exchange of ideas, the sign language plays an important role in deaf knowledge acquisition, and cognitive development and social development. So, the sign language synthesis is very important and it translates the text sentence of natural language into the deaf sign language automatically, and displays out really through virtual human produced by a computer. And this paper to establishing a three dimensional model of the human body by using the application of VRML human body modeling technology, realizes the virtual person sign language display by a algorithm of the context related virtual human gestures action smoothing algorithm.

\section{SIGN LANGUAGE SYNTHETIC RELATED METHODS AND TECHNIQUES}

Wherever Times is specified, Times Roman or Times New Roman may be used. If neither is available on your word processor, please use the font closest in appearance to Times. Avoid using bit-mapped fonts if possible. True-Type 1 or Open Type fonts are preferred. Please embed symbol fonts, as well, for math, etc.

\section{A.VRML}

VRML[1] is a king of Virtual Reality Modeling Language which mainly used for expressing and sharing 3D virtual scene and virtual objects on Internet, it is a kind of file format describing interactive 3D object and world[2][3]. VRML describes the virtual human exclusively through the H-Anim standard [4], which divides whole body into the gravity center of the human body, 77 joints and 47 bone segments. The skeletal segments of virtual human are connected by joints, the gravity center of the human body, each skeletal and joint motion will affect state of the other node which associates with him.

The hierarchical structure of H-Anim standard achieves by the nested joint nodes, using the sacrum which is in the end of spine joints as the root of the skeleton structure, and thus traverses the entire skeleton structure upward and downward respectively, according to the order of joint. It organizes all joint into tree inheritance structure, each joint node is the parent node of joint node which ranked in the following.

\section{B.virtual human motion model}

\section{a)human body contour model}

The human body contour model is expressed by people's body with a group of connection plane area block and this regional block parametric movement by the joint motion constraints. As shown in figure 1, this model includes fourteen human body articulation point and nine rectangular to describe the head, torso and limbs skeleton information. By human body contour model we establish the virtual person gesture and control of the virtual human movement. However, compared with above method, the traditional sign language synthesis technology drives virtual human model by using OpenGL technology.

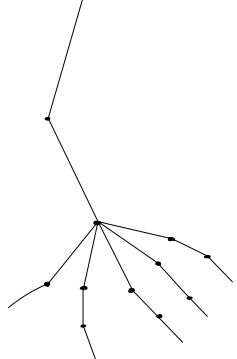

Figure1. Graph one human arm articulation point of the tree structure 


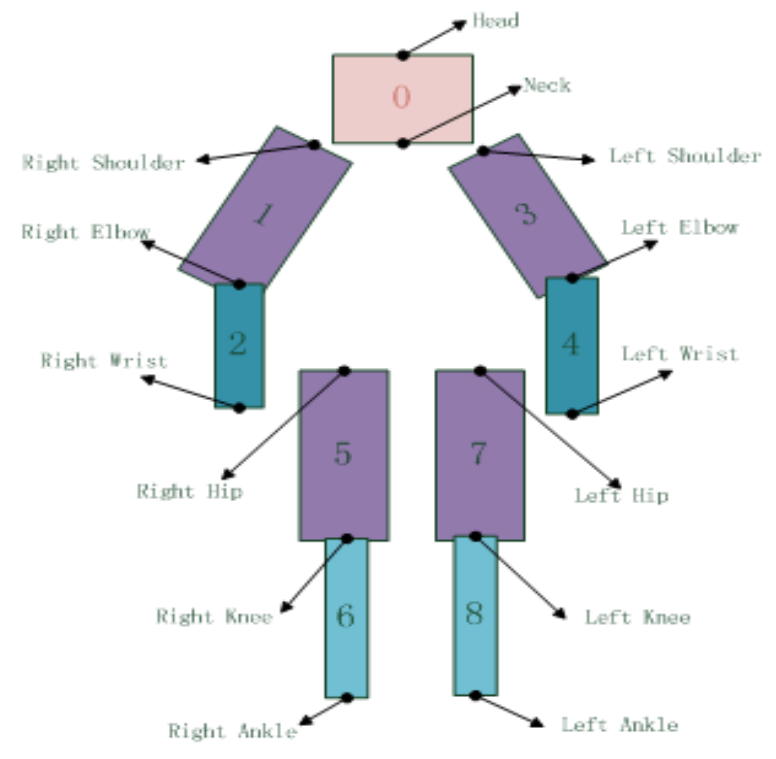

Figure 2 .human body contour model

Due to sign language is mainly synthetized by hands and arms joint coordination. So we have made the hand and arms as the research object and thereby establishing a hand and arms 'motion-control-model. Usually, shoulders, elbows, wrists and fingers joint and other kind of joints are included between in the hand and arms. As Fig. 1 shown, hand and arms' motion-control-model is described for human arm articulation point of tree structure, including black spots said each articulation point

Upper limb model based on the joint Angle of human represent the human body movement. A main joint freedom of motion of single hand and arm includes 28 points as following: three degrees of freedom of shoulder joint, a degree of freedom of elbow, three degrees of freedom of wrist joint, two degrees of freedom of the thumb wrist palm joint, two degrees of freedom of the thumb metatarsophalangeal joint, a degree of freedom of thumb articulatory, two degrees of freedom of the remaining four refers to the metatarsophalangeal joint from each, and a degree of freedom of the remaining four finger joints between each.

\section{b)Arm motion display of the human body model}

Human arm motion system is realized and run in VS2008 and OpenGL software environment and displayed by the control point, the line, and the body. First of all, it displays the point model of motion of control point, then the linear display, and finally form a body model of a human motion display.

\section{C.The construction of hand repertoire}

According to the characteristics of Chinese sign language and the multimedia technology through the software platform, we construct and synthetize Chinese sign language gestures library on the computer.At last we can check effect through the VRML model of the human body in Sign language gestures library and are not satisfied with gestures effect of modification.

In the final result, the sign language including continuous gestures make using of the keys frame identifying method that is discriminating the key frame which can best embody the sign language action essential characteristics in the state of gestures to form relatively accurate sign language.

D.Context-sensitive gestures movement smoothing algorithm

\section{a)Basic idea}

The smoothness of Virtual human gestures movement will directly affect the gestures motion expression and understandability .However, the virtual person gesture movement [5] [6] is a animation sequence joined by some Yuan animation data together and there are a lot difference of gestures action

In the adjacent two hand words or the same hand in the different root words. So, gesture movement in adjacent root cohesion place may have a amplitude of variation without smooth processing Though we need to add a certain amount of key frame in the place of cohesion when we do the sign language synthesis for the Strict technical requirements in of the animation image quality of virtual human ,soft and smoothness degree of gestures action in the actual process of sign language expression, if the number overmuch, it will inevitably leads gesture action to become slow and is short of smooth effect oppositely. Thus the number of frames to addition must meet standards

Which can measure two frame "distance" to achieve uniform gestural smooth transition.

\section{b)movement smoothing algorithm based on context gestures}

Studies show that the rotation Angles change by the shoulder, elbow joint mainly influence to gesture movement smoothness, so we let the shoulder, elbow joint as a center to establish direct coordinate system. The shoulder wound rotate by the three directions of $\mathrm{X}, \mathrm{Y}, \mathrm{Z}$ when elbow rotates by the two directions of $\mathrm{X}, \mathrm{Y}$ because of it do spherical motion around by the shoulder joint,. Therefore it applicants the SLERP (spherical linear interpolation) algorithm to describe the rotation movement of shoulder.

Here's a hypothetical in a process of gesture movement, point $\mathrm{p} 0$ andp1 are as four yuan arrays of two key frame corresponding to shoulder joint of the adjacent at some time, interpolation frame number $n$ is value got by the distance between $\mathrm{p} 0$ and $\mathrm{p} 1$ over threshold value $\Theta$...in interpolation first I frame ( 1 acuities I acuities $n) t=I / n+1$, it is:

$$
\operatorname{Slerp}(\mathrm{p} 0, \mathrm{p} 1 ; \mathrm{t})=\frac{\sin (1-t) \Omega}{\sin \Omega} p_{0}+\frac{\sin t \Omega}{\sin \Omega} p_{1}
$$

The $\Omega$ is rotation Angle, $\cos \Omega=p_{0} \cdot p_{1}$ 


\section{DESIGNING AND IMPLEMENTATION}

\section{A.System Framework of Sign Language Synthesis}

The main body of the system framework as shown in Figure 3

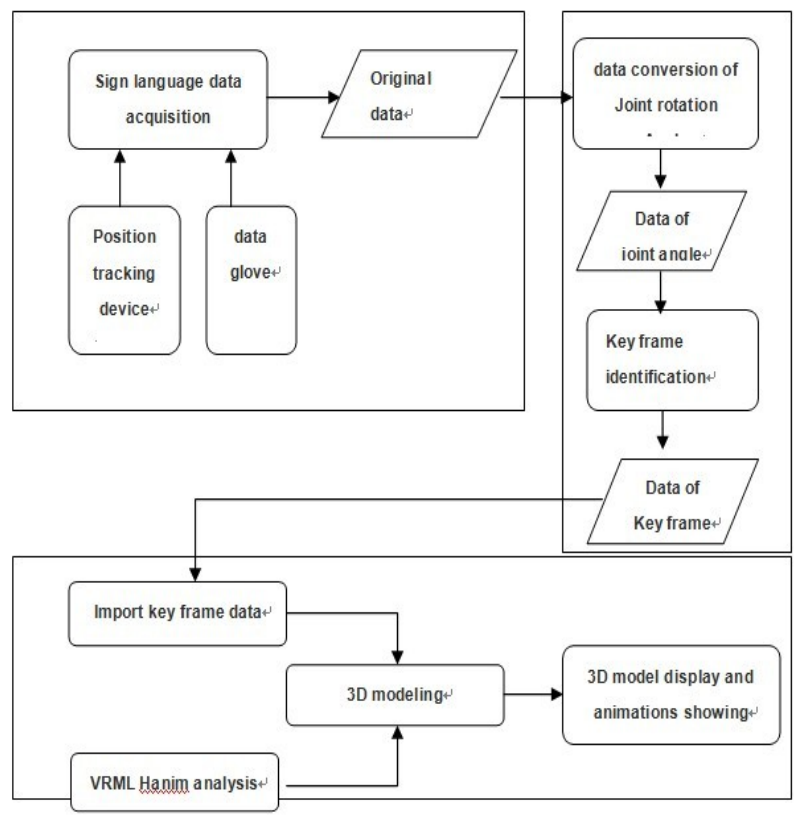

Figure. 3 the main body frame of the sign language synthesis system

\section{B.the key technology of sign language synthesis}

An establishment of sign language synthesis system includes: data collection, data conversion, data display. However Sign language expression and the final real effect mainly consists of the following four influences:

(1) Detailed sign language action information acquired by the hardware equipment in real time and including space position and direction of rotation of arms and hands

(2) The position of the human upper limb joints and the rotation Angle of joint point in relative coordinate system calculated by the original data

(3) Data of joint angles format motion curves according to different joint, and extract the key frame data representing the action.

(4) According to the key frame data to generate 3D virtual human animation automatically.

\section{RESULTS AND ANALYSIS}

Experiment done in this paper needs the configuration of hardware and software as following: CPU for P4 3.0 GHZ, memory for $2 \mathrm{~GB}$, the operating system for Windows $\mathrm{XP}$, and programming language $\mathrm{C}++$.

Through above algorithm we smoothed the last frame of Chinese text "da jia hao", means "hello" in English, and the first frame in the same phrase .Finally, we compared our results with the original Chinese sign language synthesis system, as Figure 5 shown.

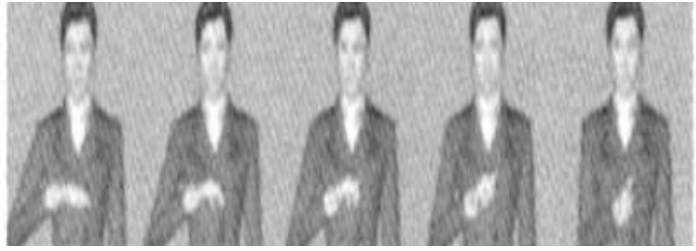

(a) the result of our method

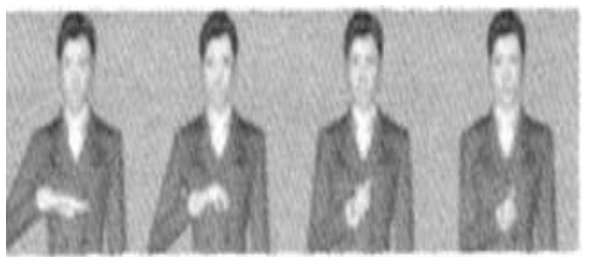

(b) the result of original Chinese sign language synthesis system

Figure 4 the comparison of original Chinese sign language synthesis system and our method of signing language synthesis system

The algorithm of sign language synthesis proposed in this paper firstly calculated an average threshold, through the training sample space statistical learning, then compared the two distance between frame which is less than the threshold value or not, that is, to find the approximate constraint relations of moving spherical minor arc length in the process of the rotation angle of elbow joint rounding the $\mathrm{X}$ direction with spherical motion of the elbow (expressed by three rotation Angle and the variation of the shoulder joint ) through the statistical learning methods. If compared results is small that we can think change amplitude of gestures movement as be small enough, and determine whether need interpolate frame or not, so as to realize smooth transition gestures sports uniform.

\section{CONCLUSION}

Building the virtual human body model based on VRML is the premise of the synthesis of sign language and the movement smoothing algorithm based the context gestures carries on the key frame interpolation to make the virtual people sign language expression more realistic, sign gestures more accurate.

\section{REFERENCES}

[1] ISO/IEC DIS 14772, VRML97 Specification [S].

[2] Stephan Diehl.VRML [J]. Informatik-Spektrum. Vol.20,1997(5): 294-295.

[3] Zhaoqi.Wang, Changshui.Yang, Wen.Gao.. Based on VRMLof the chinese sign language in $3 \mathrm{D}$ display[J]. Journal of computer research and development, 2003,40 (3) : 459-463.

[4] Zhiguo.Lu, Yan.Li, Hangen.He. Based on the Poser model of 3D human body modeling method $[\mathrm{J}]$. Computer Engineering, 2008, 34( 13) : $256,257,261$.

[5] Jintae Lee K.Model-Based Analyais of Hand Posture[J].IEEE Computer Graphies and Application, 1995(9):77-86.

[6] Cavazza M, Earnshaw R, Thalmann N.M. Motion Control of Virtual Humans.IEEE Computer Graphics and Applications.1998, 18(5):24 31. 